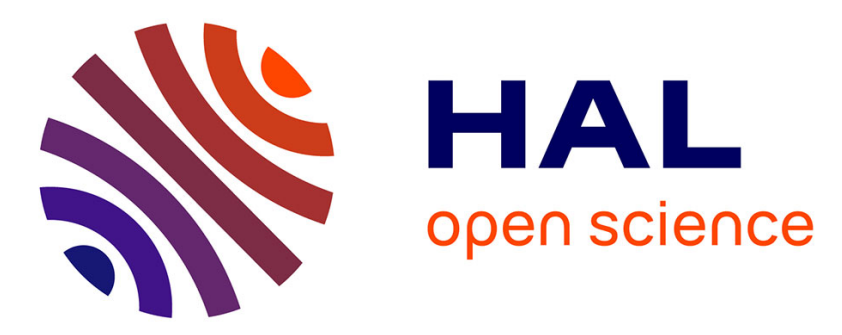

\title{
Internal waves generated by a moving sphere and its wake in a stratified fluid
}

\author{
E. Hopfinger, J. -B. Flor, J. -M. Chomaz, P. Bonneton
}

\section{To cite this version:}

E. Hopfinger, J. -B. Flor, J. -M. Chomaz, P. Bonneton. Internal waves generated by a moving sphere and its wake in a stratified fluid. Experiments in Fluids, 1991, 11 (4), pp.255-261. 10.1007/BF00192753 . hal-02140429

\section{HAL Id: hal-02140429 \\ https://hal.science/hal-02140429}

Submitted on 23 Mar 2021

HAL is a multi-disciplinary open access archive for the deposit and dissemination of scientific research documents, whether they are published or not. The documents may come from teaching and research institutions in France or abroad, or from public or private research centers.
L'archive ouverte pluridisciplinaire HAL, est destinée au dépôt et à la diffusion de documents scientifiques de niveau recherche, publiés ou non, émanant des établissements d'enseignement et de recherche français ou étrangers, des laboratoires publics ou privés. 


\title{
Internal waves generated by a moving sphere and its wake in a stratified fluid
}

\author{
E. J. Hopfinger and J.-B. Flor* \\ Institut de Mécanique de Grenoble, B.P. 53, F-38041 Grenoble, France
}

J.-M. Chomaz and P. Bonneton

Météorologie Nationale, CNRM Toulouse, 42 Ave. Coriolis, F-31057 Toulouse, France

\begin{abstract}
The internal gravity waves and the turbulent wake of a sphere moving through stratified fluid were studied by the fluorescent dye technique. The Reynolds number $\operatorname{Re}=U \cdot 2 a / v$ was kept nearly constant at about $3 \cdot 10^{3}$ and the Froude number $F=U / a N$ ranged from 0.5 to 12.5 . It is observed that waves generated by the body are dominant only when $F<4$ and are replaced by waves generated by the large scale coherent structures of the wake when $F>4$.
\end{abstract}

\section{Introduction}

Moving bodies in a stratified fluid at large Reynolds number generate internal waves by their movement, referred to as lee waves, as well as by the turbulent wake. Theoretical treatment has been generally limited to waves generated by moving point sources (Lighthill 1978; Miles 1971). Comparison with experiments of these theories, performed with bodies of revolution, has focused on the shape of the phase lines obtained by the schlieren technique. Good qualitative agreement was found some distance downstream of the obstacle and outside the wake region (Peat and Stevenson 1975; Makarov and Chashechkin 1981; Chashechkin 1989). Attempts to include in the theory the finite dimension of the moving body have also been made (Long 1955; Crapper 1959; Smith 1980; Gorodtsov and Teodorovich 1982; Janowitz 1984) and a detailed study of the flow structure and the waves in the lee of surface mounted obstacles, representing three-dimensional hills, has also been made by Hunt and Snyder (1980) and Castro et al. (1983). The flow structure and wave field of bodies of revolution at low Reynolds number is accessible by numerical simulation as was beautifully demonstrated by Hanazaki (1988) who simulated the flow field of a sphere at $R e=200$ moving in a linearly stratified fluid.

Little is available on the effect of a turbulent wake. Only Gilreath and Brandt (1985) seem to have studied the lee

\footnotetext{
* Present address: Institute of Metereology and Oceanography, University of Utrecht, P.O.B. 80.005, 3508 Utrecht
}

waves and also the short, random internal waves produced by a self-propelled and towed elliptical body and its wake. The emphasis was placed on the wave amplitude. Lin and Pao (1979) considered the conditions of the collapse of the wake of streamlined bodies, selfpropelled or not. Of interest in this context is also the collapse of highly turbulent regions created for instance by an oscillating grid in a stratified flow (Merritt, 1974).

Research on the wake and wave field of streamlined bodies of revolution, selfpropelled in particular, in a stratified fluid is motivated by direct practical applications. However, for the understanding of wake collapse conditions and of the gravity wave field generated by the body and the turbulent wake, the spherical geometry is of interest because the sphere is a reference case of bodies of revolution.

In the present paper we present novel visualisations of the wake structure and the internal wave field generated by a moving sphere and its wake in a stratified fluid. The Reynolds number was sufficiently large $\left(\operatorname{Re}=U 2 a / v \simeq 3 \cdot 10^{3}\right)$ for the wake to be fully turbulent in homogeneous fluid. The Froude number $F=U / a N$, where $U$ is the velocity of the sphere, $a$ its radius and $N=\left(-g / \varrho_{0} \mathrm{~d} \varrho / \mathrm{d} z\right)^{1 / 2}$, was varied between about 0.5 and 12.5. The interesting aspect pointed out in the present paper is that the flow field is dominated by lee waves at low Froude number and by random waves at large values of $F$.

\section{Experiments}

The experiments were mainly conducted in a translucent towing tank $50 \mathrm{~cm}$ wide, $40 \mathrm{~cm}$ deep and $6 \mathrm{~m}$ long. A hard, hollow plastic sphere of radius $a=2.5 \mathrm{~cm}$ and nearly the same density as water, was towed horizontally through the fluid by an endless, $1 \mathrm{~mm}$ thick, steel cable. In order to prevent oscillations or rotation of the sphere, three stationary guiding wires, $0.8 \mathrm{~mm}$ thick, were stretched along the tank. The sphere touched these wires at its periphery. In most of the experiments the velocity was close to $6 \mathrm{~cm} / \mathrm{s}$, giving a Reynolds number $R e=3 \cdot 10^{3}$, implying a subcriti- 
cal turbulent wake regime. The sphere was positioned $18.3 \mathrm{~cm}$ above the bottom of the tank and the water depth was $36 \mathrm{~cm}$. For the stratified runs a linear salt stratification was established by the two-tank filling technique. The buoyancy frequency $N$ was varied between 0.6 and $1.9 \mathrm{rad} \mathrm{s}^{-1}$, giving $1<F<4.3$, where $F=U / a N$ is the Froude number based on the radius of the sphere. Lower and larger Froude numbers were obtained by lowering or increasing the velocity of the sphere.

When towing the sphere by an endless cable, the boundary layer which develops on it may cause some flow disturbances. We are not aware of theoretical work giving the laminar boundary layer growth on a moving semi-infinite rod. An approximation of the thickness can be obtained by momentum balance and this gives, for the present experimental conditions, a thickness in front of the sphere of the order of the wire diameter. Shadowgraph observations confirmed these calculations. The effect of this boundary layer on the flow field around the sphere was thus negligible. Another problem could arise from flow confinement by the proximity of the bottom and the free surface and also the side walls. Wave reflection from these walls could modify the wave field. A criterion for wave reflection from the bottom and the top (a free surface like a rigid wall for wave reflection) to remain unimportant is that the Froude number $F_{D}=U / D N<1 / \pi$ (Aksenov et al. 1989), where $2 D$ is the fluid depth. The influence of the side walls is less well documented. The requirement is that the width is one to two times the depth (Graham and Graham 1980).

In order to be sure that confinement effects remained indeed negligible when $F_{D}<1 / \pi$, we conducted some experiments in the large tank of the CNRM, $100 \mathrm{~cm}$ deep, $300 \mathrm{~cm}$ wide and $22 \mathrm{~m}$ long. In particular, the large Froude number internal wave field was obtained in this tank. A different towing technique was also used. The sphere, of $2.5 \mathrm{~cm}$ radius, was ballasted with lead and suspended on three steel wires $0.1 \mathrm{~mm}$ thick (Chomaz et al. 1990). When $F_{D}$ was less than $1 / \pi$, the results obtained in the big tank were identical to those of the small tank.

The flow visualisations were made by the fluorescent dye technique. For the visualisation of the wake itself, fluoresceine was introduced in the sphere before the start of each experiment, and when the sphere was set into motion it reached the surface of the sphere through circumferentially placed small holes. The dye was made visible by a laser sheet about $2 \mathrm{~mm}$ thick. For the visualisations of the internal wave field, horizontal dye sheets were introduced by pulling thin cotton threads, soaked with fluorescent dye, slowely through the fluid. The Reynolds number was kept very small and no disturbances of the stratification were introduced. When intersected by a vertical laser sheet these dye sheets appeared as vertically spaced, horizontal colour lines. When one of the dye sheets was illuminated by a horizontal laser sheet a fringe pattern appeared when the plane was deformed by internal waves. No noticeable optical distortions of the dye lines due to index of refraction variations were detected. Outside the wake internal wave motions cause only small density variations.

\section{Experimental results}

\subsection{Qualitative observations}

A knowledge of the wake structure in homogeneous fluid is essential to the interpretation of the stratification effect. Figure 1 shows a visualisation of the wake structure in homogeneous fluid for a Reynolds number $R e=3 \cdot 10^{3}$. This figure represents a central slice, about $2 \mathrm{~mm}$ thick, of the wake marked by dye injected at the surface of the sphere and intersected by the laser light sheet. The dye released on the surface marks the boundary layer on the sphere and hence the vorticity containing fluid which then separates from the sphere. Downstream of separation the dye gives a qualitative view of the vortex shedding and the spiral formation. In particular, it is seen that near the sphere there exists an attached recirculating region, one to two sphere diameters in length, with a cylindrical vortex sheet known from previous studies of the wake of spheres (Achenbach 1974; Pao and Kao 1977). On this vortex sheet develops a KelvinHelmholtz instability followed by a roll-up into vortex rings which are then shed at the downstream end of the recirculating region. A complicated but fairly regular spiral vortex pattern emerges. Two topologies of this pattern have been

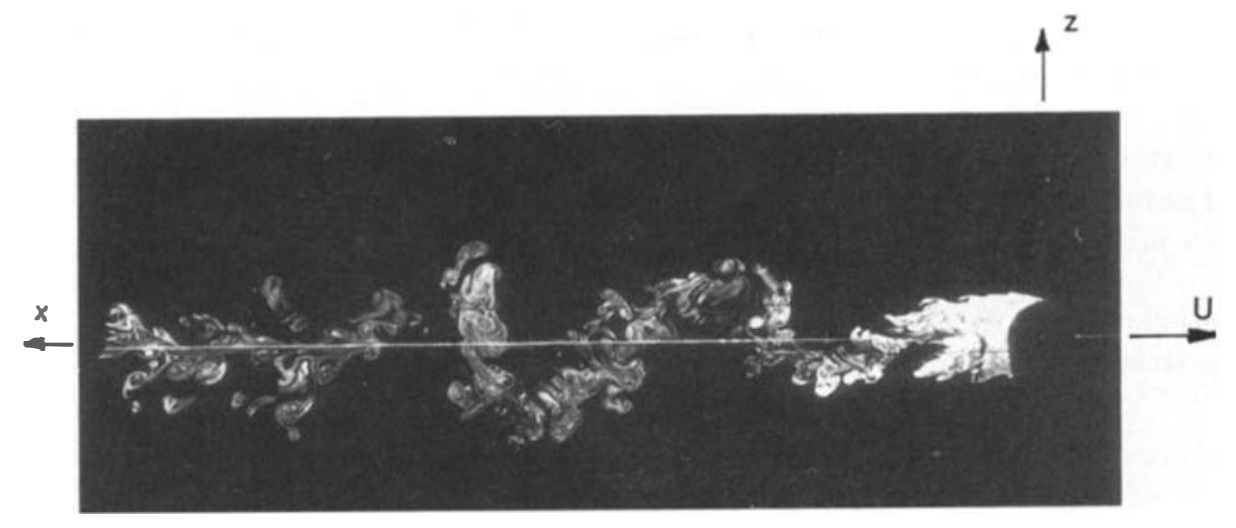

Fig. 1. The turbulent wake in homogeneous fluid at $R e=3 \cdot 10^{3}$. Fluorescent dye is introduced on the surface of the sphere and the wake is visualised by a $2 \mathrm{~mm}$ thick laser sheet in the central plane, $y=0$. The scale is given by the sphere radius 


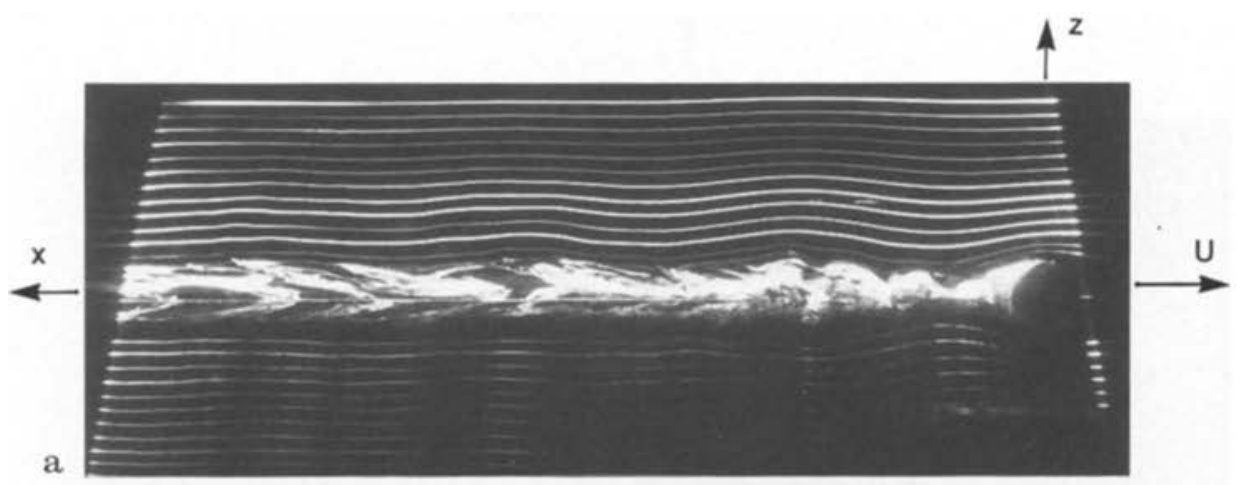

b

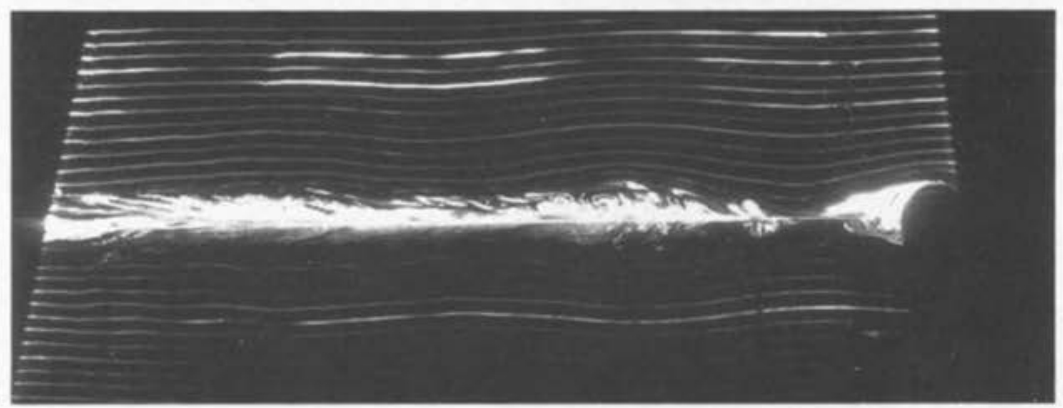

c

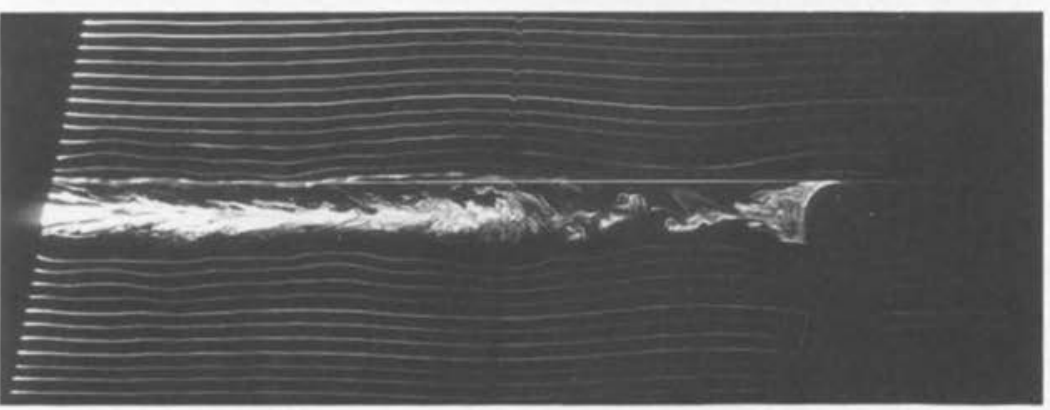

d

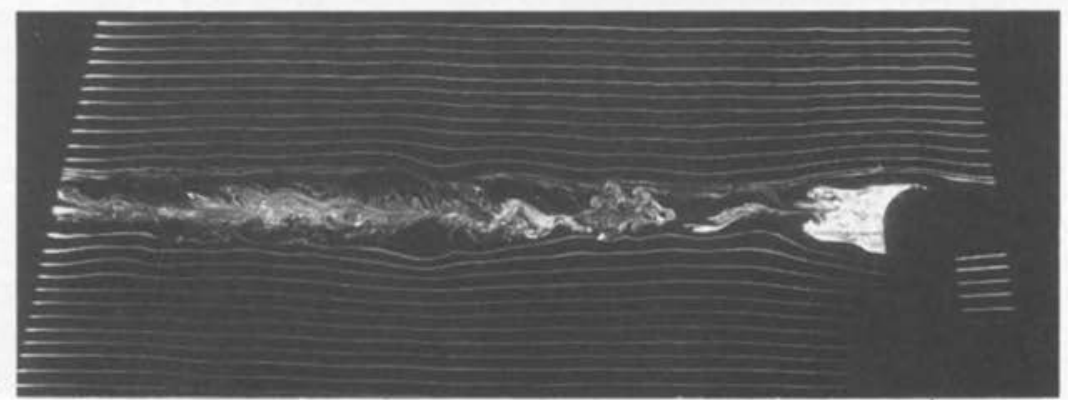

Fig. 2a-d. Visualisation of a vertical section, $y=0$, of the wake and the internal gravity wave field, predominantly lee waves, in stratified fluid. a, $F=1.2$, $N t=22.6 ; \mathbf{b}, 1.5,17.4 ; \mathbf{c} 1.7,13.7$; d, $2.5,10.2$. The non-dimensional times $N t$ are measured from the center of the sphere and refer to the left hand side of the photographs. The scale is given by the sphere diameter equal to $5 \mathrm{~cm}$. Re is $3 \cdot 10^{3}$ proposed: a double helix pattern proposed by Pao and Kao and a more complicated "handle" pattern by Achenbach. The double helix structure is seducing because it has a smooth transition to a Karman vortex street when the obstacle is elongated in the spanwise direction. The image shown in Fig. 1 is however difficult to interpret in terms of such a structure. The abrupt change in the vortex orientation seen in Fig. 1 is more characteristic of the structure proposed by Achenbach. Here, we are mainly interested in the radial excursions of these helical structure and the associated Strouhal number which is $S t=f d / U \simeq 0.2$, nearly indepen- dent of Reynolds number. The Strouhal number associated with the Kelvin-Helmholtz mode is strongly dependent on Re.

The stratification effect on the wake is shown in Figs. $2 a-2 d$. In these figures the Reynolds number is the same as in Fig. 1 and the Froude number is, respectively, 1.2, $1.5,1.7,2.5$. The scale in these photographs is given by the sphere diameter. It is clear from these figures that waves generated by the moving sphere dominate the flow when $F<2.5$ and can be clearly identified as long as $F<4$. The wave pattern is very similar to the one obtained by Hanazaki 


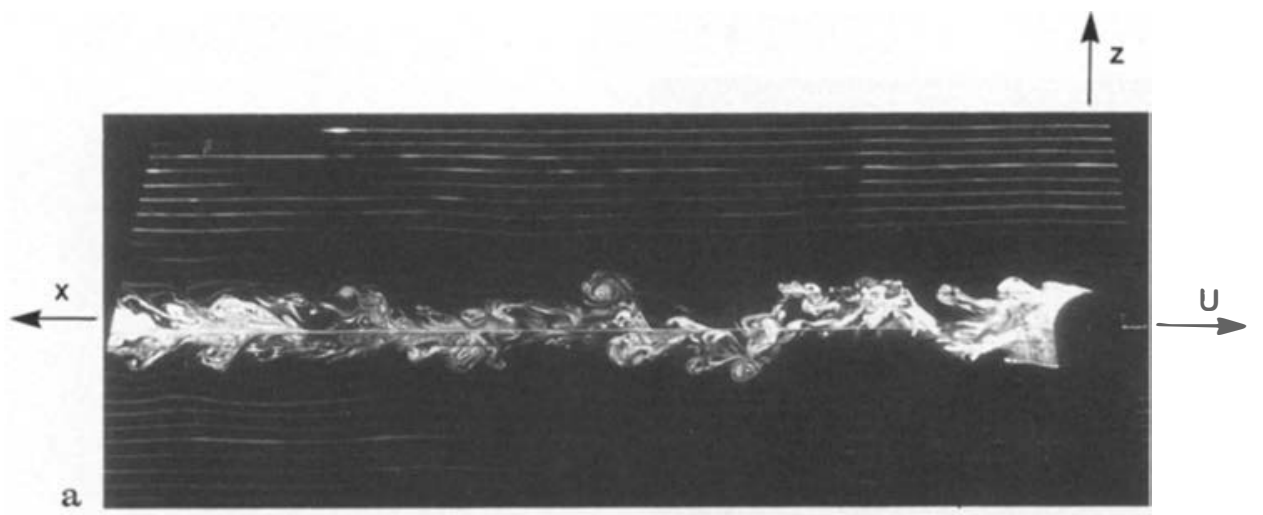

a

b

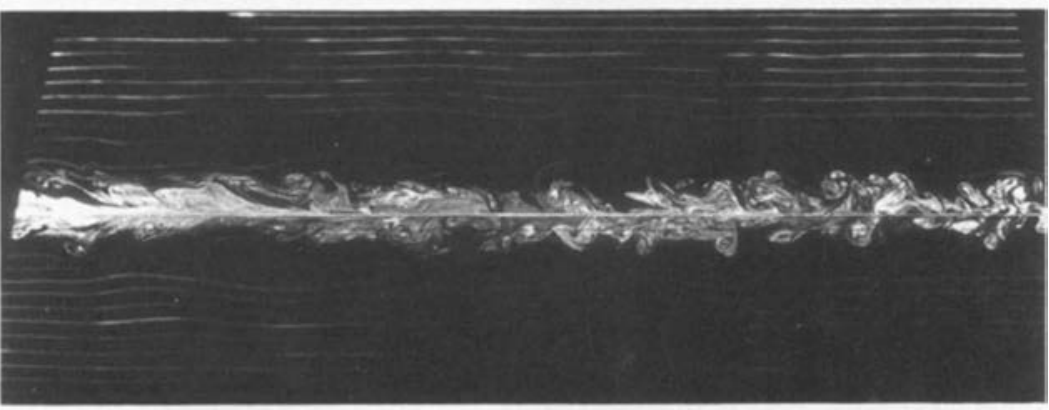

c

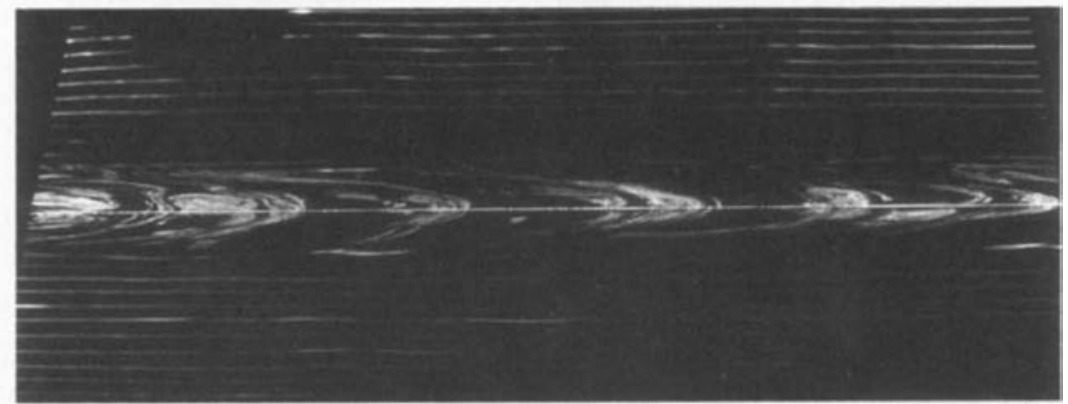

Fig. 3a-c. Visualisation (section $y=0$ ) of the internal gravity waves for $F=4.3$ at different times $N t$. a, $N t=6.6 ; \mathbf{b}, \quad N t=2.2$ to $9.5 ; \mathbf{c}$, $N t=65.6$ to 72.9 . Re and the scale are as in Fig. 2
(1988) for $R e=200$. The turbulent wake is completely suppressed when $0.4<F<1$. Below $F=0.4$ the wake has a quasi-twodimensional structure because of interference of the waves with the body. When $F>1$, random waves of shorter wavelength are generated by the turbulence in the wake, but their amplitude remains negligibly small when $F<2.5$. At larger Froude numbers these random internal waves are the dominant ones. This is shown in Fig. 3 a to $3 \mathrm{c}$, corresponding to a Froude number of 4.3 , where the only wave field clearly visible is of short wavelength. The deformations of the dye lines which are isodensity lines are wholly due to waves generated by the body and the wake because no other outside disturbances are present except for occasional air bubbles which rise through the fluid. Wave amplitudes as small as $0.1 \mathrm{~mm}$ can, therefore, be detected.

The wake shown in Fig. $3 \mathrm{a}$ is, in the very near field, similar to the neutral wake structure. When compared with Fig. 1 it appears that the wake growth begins to be affected by stratification at a location of about $20 \mathrm{~cm}$ downstream of the sphere which corresponds to a nondimensional time $N t=2.0$. The spiral structure is visible up to a time $N t=3.4$ though its vertical amplitude is less than in the neutral wake. Figure $3 \mathrm{~b}$ shows the wake between $N t=2.5$ and 9.5, and this figure illustrates that overturning of turbulent eddies in the wake continues to exist at smaller and smaller scales until $N t \simeq 9$. Overturning gives progressively way to striations and ceases completely when $N t=13$ to 14 . These time scales are comparable with those characteristic of the collapse of oscillating grid turbulence (Hopfinger, 1987). Figure $3 \mathrm{c}$, where the nondimensional time is $N t=70$, shows the fully collapsed wake which has a curious "hairpin" shaped dye pattern. This pattern begins to take shape at about $N t=19$ when $F=4.3$ and slightly earlier when $F$ is lower $(N t=16$ when $F=1.2$ ). In the horizontal plane the wake for large $N t$ has a quasi two-dimensional turbulence structure (see Fig. $8 \mathrm{c}$ of Hopfinger (1987) or Fig. 1 of Pao and Kao (1977)). It is seen from these figures that dye accumulates in bands and vortices and when intersected by a vertical sheet would 
show up as quasi-periodic dye patches. A more detailed study of this far wake is required to establish the correspondence between the observed structures in the two planes.

\subsection{Interpretation of the waves generated by the body in terms of linear theory}

The dispersion relation for linear waves is (Lighthill 1978)

$\omega^{2}=N^{2}\left(\boldsymbol{k}_{H} / \boldsymbol{k}\right)^{2}$,

where $\boldsymbol{k}_{H}$ is the horizontal component of the wave vector $\boldsymbol{k}$. From (1) it is readily found that the group velocity $\boldsymbol{v}_{G}=\mathrm{d} \omega /$ $\mathrm{d} \boldsymbol{k}$ is orthogonal to the phase velocity $\boldsymbol{v}_{\Phi}=\omega / \boldsymbol{k}$. The magnitudes of the group and phase velocities are, respectively,

$v_{G}=(N / k) \sin \theta$

$v_{\Phi}=(N / k) \cos \theta$

where $\theta$ is the angle between the wave vector and the horizontal. The Doppler frequency in the frame fixed in the fluid is $\omega_{r}=\omega_{0}+\boldsymbol{k} \cdot \boldsymbol{U}$ (Lighthill 1978), so that, in the frame moving with the source, $\omega_{0}=\omega_{r}-\boldsymbol{k} \cdot \boldsymbol{U}$. In the present case $\omega_{0}=0$, hence $\boldsymbol{v}_{\Phi 0}=0$, indicating that the phase lines are stationary with respect to the body. Here $\boldsymbol{U}$ is taken positive in the direction of source displacement which is opposite to the $x$ direction. In the plane $y=0$ the phase velocity is thus simply $v_{\Phi}=U \cos \theta$. This fixes the wavelength in this plane to be $\lambda=2 \pi U / N$, or in terms of the Froude number

$\lambda / 2 a=\pi F$.

The surfaces of constant phase are given by $\boldsymbol{k} \cdot \boldsymbol{X}=\phi$ (Lighthill 1978) with $X=X \boldsymbol{n}$ and $X=(x, y, z)$. Since $\boldsymbol{k} \cdot\left(\boldsymbol{v}_{G}-\boldsymbol{U}\right)=-\boldsymbol{k} \cdot \boldsymbol{U}$ and $\boldsymbol{n}$ is colinear with $\left(\boldsymbol{v}_{G}-\boldsymbol{U}\right)$, the relation $X / \phi=n / \boldsymbol{k} \cdot \boldsymbol{n}$ can be written in the form

$\boldsymbol{X} / \phi=-\left(\boldsymbol{v}_{G}-\boldsymbol{U}\right) / \boldsymbol{k} \cdot \boldsymbol{U}$.

Substitution of the expression for $\boldsymbol{v}_{G}$ and scaling $X$ by $X^{*}=X N / \phi U$, the equation of the three-dimensional phase surfaces is obtained from (5) in the form (see, e.g., Makarov and Chashechkin, 1981)

$x^{* 2} /\left(1-z^{* 2}\right)-y^{* 2} / z^{* 2}=1$.

In Figure 4 we have retraced the isopycnal lines for $F=1.2$ from Fig. $2 \mathrm{a}$. The phase lines in a vertical central plane $y=0$, determined from linear theory, Eq. (6), are shown by the dashed semi-circular lines. These lines pass within experimental error through the positions of maximum amplitude of the waves. The observed phase line pattern in a horizontal plane, $4.5 \mathrm{~cm}$ above the center of the sphere $(z / a=1.8)$, is shown in Fig. 5 for a Froude number $F=1.6$. The phase lines calculated from linear theory, Eq. (6), are also included in this figure. There is good agreement except for some perturbations of the experimental phase lines by the turbulence in the wake.

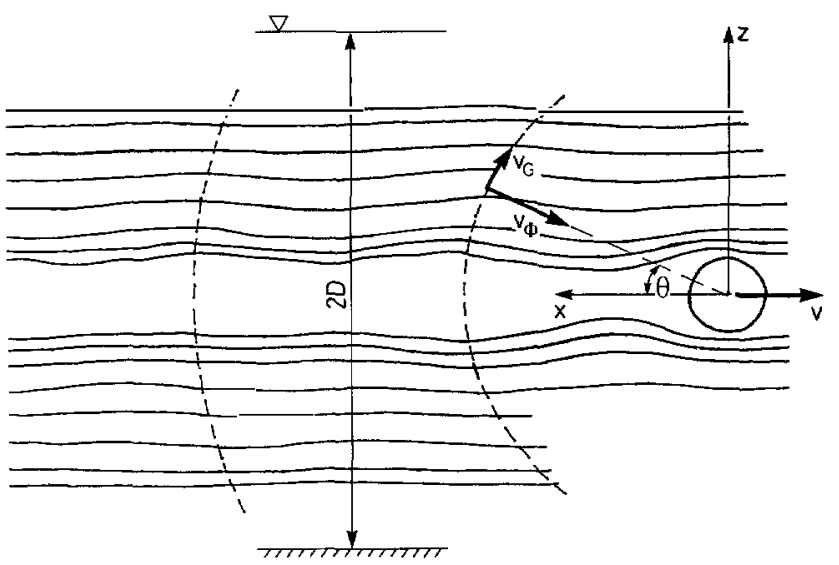

Fig. 4. Isopycnal lines (solid lines) for $F=1.2$ retraced from Fig. 2 a and theoretical phase lines (dashed lines) in a vertical plane, $y=0$

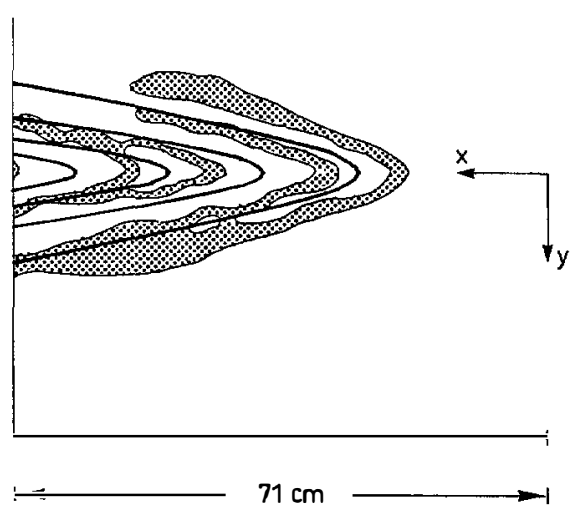

Fig. 5. Phase lines (middle of clear zones) in a horizontal plane $z=4.5 \mathrm{~cm}$ above the centre of the sphere, located at $x, y, z=0$, and comparison with linear theory (solid lines). The phase line spacing is $\lambda / 2$

In Fig. 6 the measured non-dimensionalised wavelengths are plotted as a function of Froude number. It is seen that agreement with linear theory is very good when $F<4$. When $F>4$, shorter wavelength waves, generated by the coherent structures of the turbulent wake, dominate and lee waves can no longer be detected. The observation of this transition from one wave regime to another is an interesting result. This is not a confinement effect. For two-dimensional bodies there exists a cut-off Froude number $F_{D}=1 / \pi$ (Long 1955; Aksenov et al. 1985) for the existence of lee waves. In the three-dimensional case, however, lee waves exist also when $F_{D}>1 / \pi$, only the phase lines are changed (Aksenov et al. 1989). In any event, the observed transition was independent of $a / D$ which was varied by a factor of about three in our experiments, namely $0.14,0.1$ and 0.05 . As discussed below, the observed transition shown in Fig. 6 is rather attributed to the waves produced by the turbulence of the wake which are dominant when $F>4$. 
Linear theary

00000 Lee waves in o vertical plone

Mrd Lee waves in a horizontal plane

Long time woves produced

by turbulence

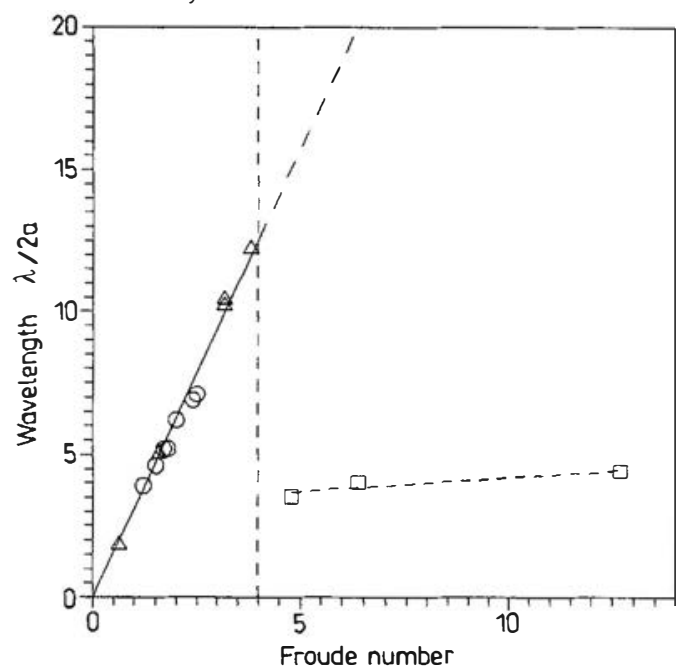

Fig. 6. Wavelengths non-dimensionalized by $2 a$ as a function of Froude number. Wavelength of lee waves determined from visualisations: $O$, in the vertical plane; $\Delta$, in a horizontal plane; $m$, waves generated by the turbulent wake

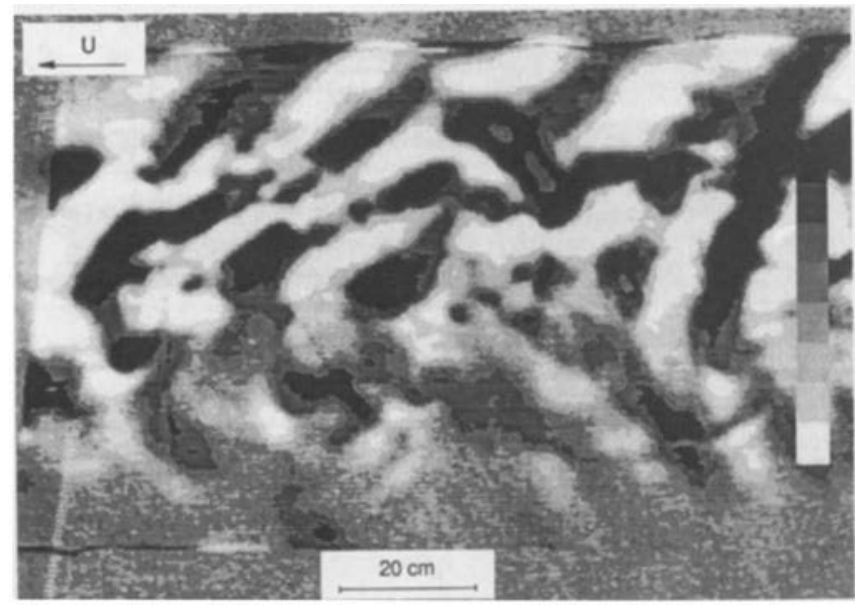

Fig. 7. Visualisation of the phase lines in a horizontal plane $z=12.5 \mathrm{~cm}$ above the centre of the obstacle for $F=12.5$ and $N t=75.4$. Image taken in the big tank $100 \mathrm{~cm}$ deep and $300 \mathrm{~cm}$ wide. The sphere is moving here from right to left

\subsection{Waves generated by the wake}

For $F>4$, the dominant wavelength of the waves generated by the turbulence in the wake is nearly independent of Froude number (Fig. 6). It is practically equal to the length of the spiral structure, approximately $8 \cdot a$, seen in Fig. $3 \mathrm{a}$, comparable with the neutral wake structure (Fig. 1). The waves are thought to be generated when these structures penetrate into the stratified fluid and then collapse or recoil on a buoancy time scale $N^{-1}$. Hence, the relative phase velocity is $v_{\Phi} / U \simeq 4 a N / \pi U=4 / \pi F$. A view of the phase lines in a horizontal plane $12.5 \mathrm{~cm}(z / a=5)$ above the center of the sphere for $F=12.5$ and $N t=75.4$ is shown in Fig. 7. This image was taken in the large tank, and therefore only about half of the wake is clearly visible because of light absorption. The predominant wavelength is the one indicated in Fig. 6, but it is clear that a Fourier analysis of this wave field would probably select additional wave lengths.

The amplitude of the waves generated by the turbulence when $F>4$ is appreciable, and lee waves, whose amplitude decreases with increasing Froude number because of an increase in turbulence pressure in the wake, disappear. When $F<4$, the spiral structure is inhibited by stratification (see for instance Fig. 2c), and the waves generated by the turbulence in this case are of very short wavelength and also of small amplitude. Lee waves remain, therefore, the dominant waves.

Waves associated with wake collapse, that is, due to a decrease in the vertical dimension of the wake, as observed by Gilreath and Brandt (1985), were not observed in the present experiments. No clear shrinkage of the wake of the sphere was in fact manifest. This is indicative of little mixing of the density field inside the wake.

\section{Conclusions}

The wake structure and the internal gravity wave field in the lee of a sphere moving through a linearly stratified fluid were studied by the fluorescent dye technique. The sphere was in the subcritical flow regime with, in general, a Reynolds number of $R e=U \cdot 2 a / v=3 \cdot 10^{3}$ and Froude numbers $0.5<f=U / a N<12.5$.

The most striking result is the observed transition from a lee wave regime in the wake of the sphere to internal gravity waves generated by the coherent turbulent structures in the wake. This transition occurs at a Froude number of about 4. The lee waves are in good agreement with linear theory of moving point sources. The random gravity waves generated by the coherent turbulent structures have a wave length of about $4 d$ which is the scale of the helical vortex structure in the wake.

Non-dimensional times, characterising the evolution of the wake itself, are also determined. From this it appears that the wake feels the stratification when $N t>2$, and collapse is completed when $N t>14$. The wake appears to be nearly two-dimensional when $N t>20$.

\section{Acknowledgements}

The authors wish to thank M. Layat of the IMG and A. Butet and M. Perrier of the CNRM for technical assistance. The work was financially supported by the DRET through contract $n^{\circ} 88-126$. One of the authors (J.-B. F.) acknowledges financial support by the French Government through contract $\mathrm{n}^{\circ} 880053$. 


\section{References}

Achenbach, E. 1974: Vortex shedding from spheres. J. Fluid Mech. 62, 209-221

Aksenov, A. V.; Kirillov, V. P.; Mozhaev, V. V.; Skorovarov, V. E.; Sheronov, A. A. 1985: Structure of internal waves in a channel. Fluid Dyn. 20, 92-96

Aksenov, A. V.; Mozhaev, V. V.; Skoroarov, V. E.; Sheronov, A. A. 1989: Phase structure of three-dimensional internal waves in a channel. Fluid Dyn. 24, 107-111

Castro, I. P.; Snyder, W. H.; Marsh, G. L. 1983: Stratified flow over three-dimensional ridges. J. Fluid Mech. 135, $261-282$

Chashechkin, Yu. D. 1989: Hydromechanics of a sphere in a stratified fluid. Fluid Dyn. 24, 1-6

Chomaz, J-M.; Bonneton, P.; Butet, A.; Hopfinger, E. J.; Perrier, M. 1990: Gravity wave patterns in the wake of a sphere in a stratified fluid. Proceedings of "Turbulence 89" (Lesieur, M. et Métais, O. Eds.)

Crapper, G. D. 1959: A three-dimensional solution for waves in the lee of mountains. J. Fluid Mech. 6, 51-76

Gilreath, H. E.; Brandt, A. 1985: Experiments on the generation of internal waves in a stratified fluid. AIAA J. 23, 693-700

Gorodtsov, V. A.; Teodorovich, E. V. 1982: Study of internal waves in the case of rapid horizontal motion of cylinders and spheres. Fluid Dyn. 17, 893-898

Graham, E. W.; Graham, B. B. 1980: The tank wall effect due to a transient vertical force moving at fixed depth in a density stratified fluid. J· Fluid Mech. 97, 91-114

Hanazaki, H. 1988: A numerical study of three-dimensional stratified flow past spheres. J. Fluid Mech. 192, 303-419
Hopfinger, E. J. 1987: Turbulence in stratified fluid: a review. J. Geophys. Res. 92, 5287-5303

Hunt, J. C. R.; Synder, W. H. 1980: Experiments on stably and neutrally stratified flow over a model three-dimensional hill. J. Fluid Mech. 96, $671-704$

Janowitz, G. S. 1984: Lee waves in three-dimensional stratified flow. J. Fluid Mech. 148, 97-108

Lighthill, J. 1978: Waves in fluids. Cambridge University Press.

Lin, J. T.; Pao, Y. H. 1979: Wakes in stratified fluids. Ann. Rev. Fluid Mech. 11, 317-338

Long, R. R. 1955: Some aspects of the flow of stratified fluids III: Continuous density gradients. Tellus 7, 341-357

Makarov, S. A.; Chashechkin, Yu. D. 1981: Apparent internal waves in a fluid with exponential density distribution. J. Appl. Mech. Techn. Phys. 22, 772-779

Merritt, G. E. 1974: Wake growth and collapse in a stratified flow. AIAA J. 12, 940-949

Miles, J. W. 1971: Internal waves generated by a horizontally moving source. Geophys. Fluid Dyn. 2, 63-87

Pao, H. P.; Kao, T. W. 1977: Vortex structure in the wake of a sphere. Phys. Fluids 20, 187-191

Peat, K. S.; Stevenson, T. N. 1975: Internal waves around a bod in a compressible density stratified fluid. J. Fluid Mech. 70, 673-688

Smith, R. B. 1980: Linear theory of stratified hydrostatic flow past an isolated mountain. Tellus $32,348-364$ 